\title{
Increasing awareness of compartment syndrome among orthopedic nurses and trauma nurse practitioners at a district general hospital: a complete audit loop
}

\author{
This article was published in the following Dove Press journal: \\ Clinical Audit \\ 30 May 2017 \\ Number of times this article has been viewed
}

\author{
Arun Khajuria \\ Rohi Shah \\ Herbert Gbejuade \\ Shabih Siddiqui \\ Trauma and Orthopaedic Department. \\ Kettering General Hospital NHS \\ Foundation Trust, Kettering, UK
}

Background: Acute compartment syndrome (ACS) is a surgical emergency defined by a critical increase in pressure within a closed osteofascial compartment requiring prompt diagnosis treatment via fasciotomy and decompression of the affected compartment. A critical factor for a poor outcome following ACS is a delay in the initial recognition and subsequent diagnosis. Orthopedic nurses (ONs) are usually the first port of call to see patients at risk of developing ACS prior to escalation. The aim of this audit project was to evaluate the baseline knowledge of the nursing staff for ACS, aiming to improve awareness and early diagnosis of the condition. Methods: A 6-point pre-course questionnaire focusing on the clinical diagnosis of ACS, early signs and symptoms, immediate interventions and complications of a delay in diagnosis was filled out by ONs to assess baseline knowledge. Following a targeted lecture, the questionnaire was repeated. A ward-based protocol was introduced for quick reference, highlighting an early escalation plan following recognition of ACS. A follow-up questionnaire was filled at 4 months. Results: A majority of the nurses involved in this audit had little or no prior clinical experience in the management of patients with ACS. Following the interventions, all staff could define ACS. Tibial shaft fractures were correctly identified as high risk for ACS ( $89 \%$ post course; $100 \%$ final questionnaire). Pain out of proportion, as well as pain on passive stretch, was correctly identified as the most important defining symptom (100\% post course; $93 \%$ final questionnaire). There was variability in the immediate response following a diagnosis of ACS, and nurses were less inclined to perform a physical intervention (splitting cast $-43 \%$ ) without prior medical review. Conclusion: Our audit highlights that simple, lecture-based interventions alongside printed ward-based protocols proved to be effective interventions. The audit emphasizes the need for continuous teaching and training of nurses to improve awareness and early diagnosis of ACS.

Keywords: orthopedics, acute compartment syndrome

\section{Introduction}

Acute compartment syndrome (ACS) is defined by a critical increase in pressure within a closed osteofascial compartment.

The critical level is the intramuscular tissue pressure, which causes the capillary bed to collapse, preventing low-pressure blood flow through the capillaries into venous drainage. ${ }^{1}$ This results in compromised tissue perfusion, ischemia and necrosis. ${ }^{2-6}$ The normal tissue pressure varies between 0 and $10 \mathrm{mmHg}$, while the capillary pressure equates to the diastolic arterial pressure. When the intramuscular tissue pressure increases and approaches the diastolic pressure, capillary blood flow reduces and
Correspondence: Rohi Shah Apartment 5D Park Rock, Castle Boulevard, Nottingham NG7 IRU, UK Tel +447878889654

Email shah.rohi@gmail.com 
ultimately ceases. The diastolic pressure needs to be at least $30 \mathrm{mmHg}$ above the intramuscular tissue pressure, otherwise compartmental capillary blood flow is obstructed, resulting in severe hypoxia to muscle and nerve tissue. ${ }^{1}$

ACS is a true surgical emergency requiring prompt diagnosis and urgent treatment via fasciotomy and decompression of all tissues within the affected compartment. ${ }^{6}$ Failure to do so may result in severe limb deformity and disability. ${ }^{6}$

A critical factor for a poor outcome following ACS is a delay in the initial recognition and diagnosis of the syndrome..$^{7-11}$ One of the main causes of a delay in diagnosis is due to insufficient awareness of this condition ${ }^{8}$ either from a nursing assessment or because of inexperience of the surgeon. Additionally, diagnoses based purely on clinical judgment may be difficult in specific cohorts of patients such as children and hypotensive patients. However, most adults who do develop ACS are not hypotensive. ${ }^{8}$ Other attributable factors that may result in a delayed diagnosis include patients who have undergone general or regional anesthesia, patients with polytrauma and concomitant soft-tissue injuries and diagnoses based on clinical signs and symptoms as opposed to pressure monitoring devices. . $^{8,12,13}$

Having a higher index of suspicion in groups of patients susceptible to ACS following patterns of injury such as tibial diaphyseal and forearm/elbow fractures would help early recognition of the condition. This would allow for immediate ward-based interventions to occur while awaiting definitive treatment in the form of fasciotomies of all affected compartments. Clinical suspicion can be reinforced by examining the patient and eliciting findings such as pain out of proportion to the injury as well as pain not eased by opioid analgesics. A delay in diagnosis and management will inevitably lead to a prolonged period of inadequate tissue perfusion and subsequently result in a poor outcome for the patient.

In the UK, orthopedic nurses (ONs) or trauma nurse practitioners (TNPs) are usually the first port of call to see patients who are at risk of developing compartment syndrome, prior to escalation to the house officer or surgeon. Therefore, it is crucial that they are able to recognize early signs of ACS, perform immediate ward-based interventions, and escalate appropriately.

With that in mind, the objective of this audit project was to evaluate the baseline knowledge pertaining to compartment syndrome of the nursing staff, with interventions aiming to improve awareness, early diagnosis and interventions where appropriate.

\section{Aims and objectives}

The aims and objectives of this audit were as follows:
1. assess the baseline knowledge of the ONs and TNPs pertaining to compartment syndrome using a tailored questionnaire;

2. identify gaps within that knowledge;

3. perform a targeted, lecture-based teaching session for the ONs and TNPs pertaining to clinical signs, symptoms and complications of ACS; commonly affected areas as well as immediate interventions and definitive treatment required;

4. reevaluate the effectiveness of this teaching session using the same questionnaire;

5. introduce a ward-based protocol for escalation of patients with suspected compartment syndrome; and

6. perform a third evaluation of the two interventions (lecture-based and ward-based protocol implementation), reassessing knowledge on awareness, diagnosis and subsequent intervention.

\section{Audit standards}

Following a targeted teaching session, all ONs and TNPs should be able to identify early warning signs for patients with suspected compartment syndrome and should be able to perform the recommended initial intervention.

\section{Methods}

A six-point multiple choice questionnaire was sent out to the ONs and TNPs ( $n=19$; Objectives 1 and 2). Although this was not a validated questionnaire, it was designed to assess core aspects of ACS and therefore deemed to be an achievable baseline for all participants at the end of the study.

The first question focused on the clinical definition of compartment syndrome, i.e., raised pressure within a compartment. Susceptible injury patterns were then assessed, i.e., identifying common areas where ACS could occur in the body (tibial and elbow fractures). Signs and symptoms were then assessed, specifically early signs and symptoms, including pain out of proportion to injury, pain on passive stretch of the muscle involved and recognizing distal sensory impairment. The questionnaire then focused on immediate response to a clinical suspicion of ACS, i.e., splitting the plaster down to skin, but leave the plaster on, and being aware that limb loss is indeed a complication of compartment syndrome.

Using results from the pre-course questionnaire and identifying aspects of ACS that were poorly understood, a target lecture was then delivered to the ONs and TNPs on 10 August 2016 (Objective 3). Of note, TNPs are differentiated from ONs with regard to higher practical skill set pertaining to orthopedic procedures, and therefore, no 
knowledge discrepancy was expected between the ONs and TNPs, specifically for ACS. Therefore, the results obtained from ONs and TNPs have been displayed collectively.

A post-course questionnaire was then filled out and assessed for any improvements in baseline knowledge (Objective 4).

Following the initial lecture-based intervention, an A4-sized ward-based protocol was introduced to all the wards (Figure 1) to provide an early escalation plan for the orthopedic staff. This protocol was based on guidelines recommended by the British Orthopaedic Association (BOA) ${ }^{14}$ and the Royal College of Nursing (RCS). ${ }^{15}$

A follow-up questionnaire was filled out by the nursing staff in December 2016, 4 months after the initial interventions to see whether the interventions had any impact on baseline knowledge and their ability to recognize and intervene where appropriate (Objective 6).

\section{Ethical approval}

As no patients were involved in the study, ethical review was not required. The audit was therefore registered and approved by the Local Audit Department of Kettering General Hospital.

\section{Results}

A total of 19 pre-course and 19 post-course questionnaires had been filled out. At 4 months, 15 complete questionnaires were completed and returned. The results from all three questionnaires have been highlighted later as a percentage value.

The first question focused on the definition of compartment syndrome. A majority of the ONs and TNPs were aware that compartment syndrome is defined by a raised pressure within a compartment ( $79 \%$ pre course vs. $100 \%$ post course vs. $87 \%$ final questionnaire; Figure 2).

The second question focused on localizing the two most common anatomical sites for ACS to occur. Most nurses (89\% pre course, $89 \%$ post course, $100 \%$ final questionnaire) were aware that tibial fractures were most commonly associated with compartment syndrome. There was some variability in the selection of the second commonest site (elbow/forearm fractures). Although ACS could theoretically occur in any compartment, only $11 \%$ of staff were aware (both pre course and post course) that elbow/forearm fractures were a common site for compartment syndrome. However, on the final questionnaire, 93\% of staff selected elbow fractures (Figure 3).

Following the lecture, all staff were aware that pain out of proportion was the most important symptom for ACS (53\% pre course vs. $100 \%$ post course). A similar picture was seen at the 4-month questionnaire (93\%; Figure 4).
There was variability in the selection of the two most important signs of compartment syndrome (pain on passive stretch and distal sensory loss) during the pre-course questionnaire. Following the lecture, all staff were aware to look for pain on passive stretch ( $42 \%$ pre course vs. $100 \%$ post course); however, there was no change in the awareness of distal sensory loss ( $42 \%$ pre course vs. $42 \%$ post course). At 4 months, there was once again variability in the selection of the most important signs, with $73 \%$ of staff selecting pain on passive stretch of the muscles and only $27 \%$ of staff selecting sensory loss (Figure 5).

With regard to the immediate response following accurate diagnosis (splitting the Plaster of Paris down to skin but keeping the plaster on), most staff thought that limb elevation would be appropriate (53\%), a counter-productive measure, and only $16 \%$ selected splitting the POP. Following the lecture, there was still a lot of variability in the answers, with only $26 \%$ selecting splitting the POP down to skin as the appropriate answer. Most staff selected escalating to the on-call doctors (32\% post course), while $21 \%$ still thought that limb elevation would be appropriate. A further $21 \%$ of staff thought that complete removal of the plaster would be appropriate. At the 4-month questionnaire, there was a slight increase in the selection of splitting the POP down to skin (16\% pre course vs. $26 \%$ post course vs. $47 \%$ final questionnaire). Limb elevation was only selected by one staff member (Figure 6).

Most staff were aware that limb loss could occur as a significant complication of compartment syndrome (74\% pre course vs. $79 \%$ post course vs. $100 \%$ final questionnaire; Figure 7).

\section{Discussion}

$\mathrm{ACS}$ is a true surgical emergency requiring early recognition, immediate ward-based interventions and definitive treatment in the form of fasciotomies of the affected compartments. The British Orthopaedic Association Standards for Trauma (BOAST) 10 guidelines (diagnosis and management of compartment syndrome of the limbs) ${ }^{14}$ and the $\mathrm{RCN}^{15}$ recommend assessment for ACS as routine evaluation of patients who present with significant limb injuries, post surgery and after prolonged surgical duration with resulting hypoperfusion of the limb. Early diagnosis is a key to preventing future complications.

A delay in diagnosis may happen because of insufficient awareness of the condition. It is therefore critical that nursing staff who look after these patients can recognize early signs of ACS, with clinical suspicion being reinforced by examining the patients. This audit project aimed to assess 


\section{Kettering General Hospital WHS}

NHS Foundation Trust

\section{Acute Compartment Syndrome (ACS)}

\section{WHAT IS COMPARTMENT SYNDROME?}

ACS is critical increase in pressure within a closed osteofascial compartment resulting in compromised tissue perfusion and potential ischaemia and necrosis. It is a true surgical emergency requiring prompt diagnosis and urgent treatment via fasciotomy and decompression of all tissues within the affected compartment.

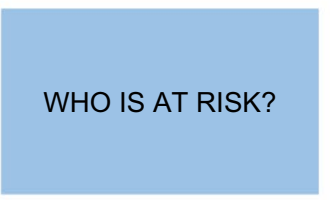

Most commonly tibial, forearm or high-energy distal radius fractures. Orthopaedic injury/intervention in patients with coagulopathies/patient taking anticoagulants.

Crush injuries.

High impact trauma, including open fractures.

Monitor hourly for the first 24 hours.

From 24 to 48 hours monitor 4 hourly.

However, if suspicions arise at any point revert to hourly monitoring.

WHAT ARE THE CLINICAL SIGNS?
Is there:
$\begin{gathered}\text { Severe Pain } \\ \text { Tense Swelling } \\ \text { Pain on stretching fingers/toes } \\ \text { Absent/delayed capillary refilling } \\ \text { (normal: }<2 \text { seconds) }\end{gathered}$

If any of the above signs are present, GO TO STEP 2

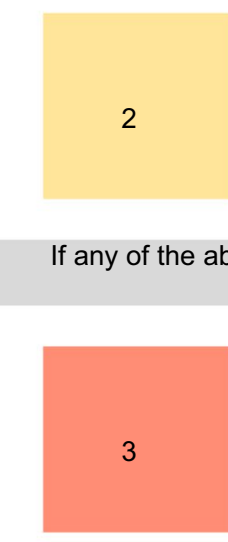

Split the POP down to skin (only split, do not remove)

Call the SHO immediately

Keep the limb elevated at heart level

Recheck limb observations in 15 mins

Guideline based on recommendations by British Orthopaedic Association and Royal College of Nursing

Call the Registrar immediately

Get the patient reviewed urgently

sent at 15 mins and the patient has not been seen by the

SHO GO TO STEP 3

Figure I Ward-based protocol for diagnosis and early intervention

Abbreviations: POP, Plaster of Paris; SHO, Senior House Office. 


\section{Compartment syndrome is defined as}

(select 1):

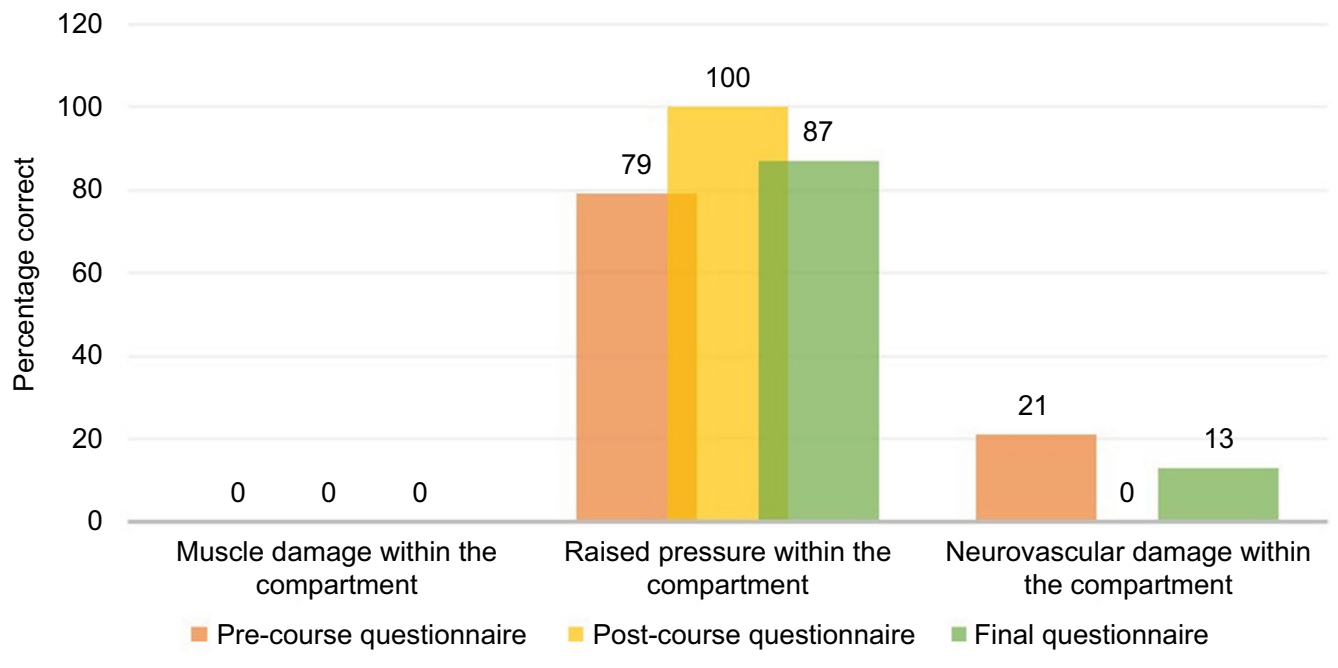

Figure 2 Question I: Compartment syndrome is defines as?

Note: Correct answer: raised pressure within the compartment.

Compartment syndrome is commonly seen following

(select 2):

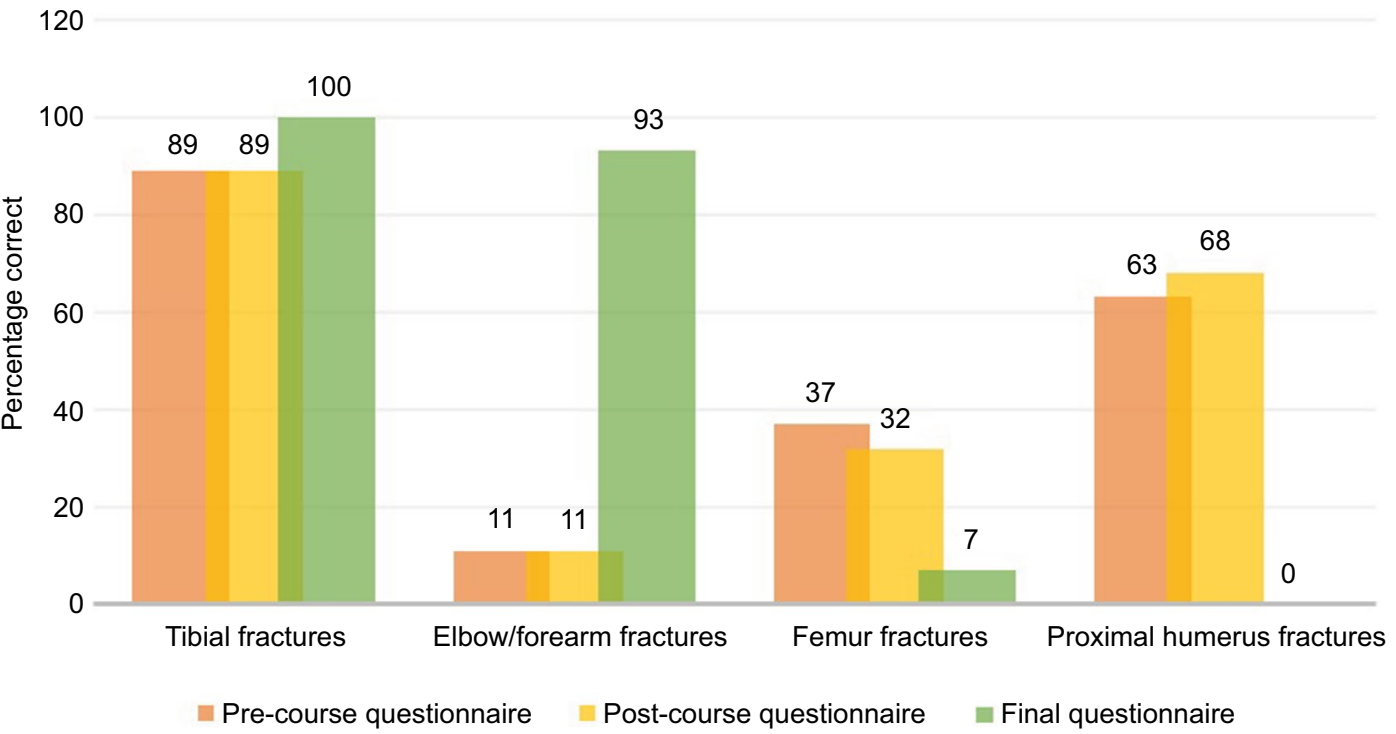

Figure 3 Question 2: Compartment syndrome is commonly seen following?

Note: Correct answers: tibial fractures and elbow fractures.

and subsequently improve their baseline knowledge. Only then can appropriate ward-based interventions be performed and subsequently escalated to the surgeon for fascial decompression.

The pre-course questionnaire highlighted key areas of poor understanding of ACS that the lecture-based intervention and implementation of the ward-based protocol attempted to address. All participants following the targeted teaching could define compartment syndrome as raised pressure within the compartment (100\%), although on reassessment at 4 months, two nurses (13\%) perceived ACS to be defined by neurovascular damage. Despite ACS theoretically occurring in any closed osteofascial compartment, it is most frequently seen in lower limb fractures and elbow/ forearm fractures. This is usually due to a higher incidence of fractures in these anatomical sites. Most ONs and TNPs correctly identified tibial fractures as high risk of ACS ( $89 \%$ post questionnaire and $100 \%$ at 4 months); however, when asked to identify the second most common area, i.e., forearm fractures, there was no change between the precourse and post-course questionnaires (11\%). A majority of staff still believed that there was a higher incidence of 
The most important symptom of compartment syndrome is (select 1):

120

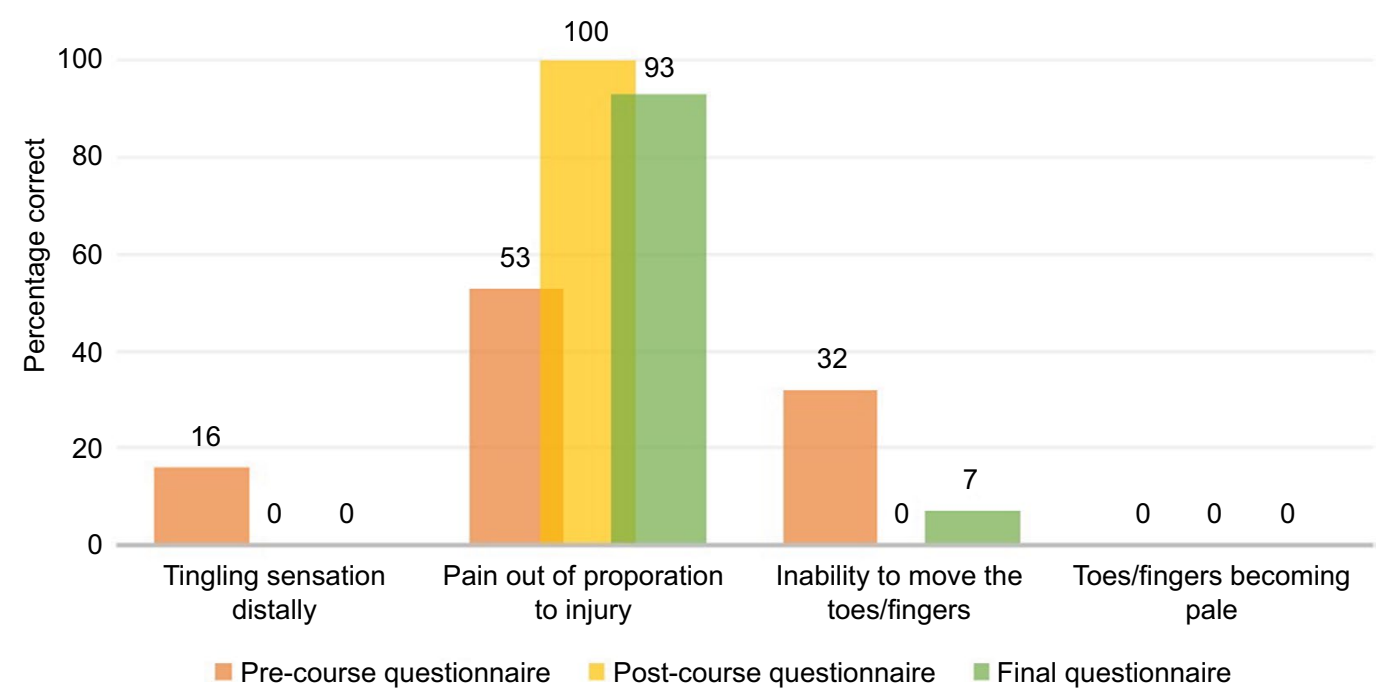

Figure 4 Question 3: The most important symptom of compartment syndrome is? Note: Correct answer: pain out of proportion to injury.

The two most important signs of compartment syndrome include (select 2):

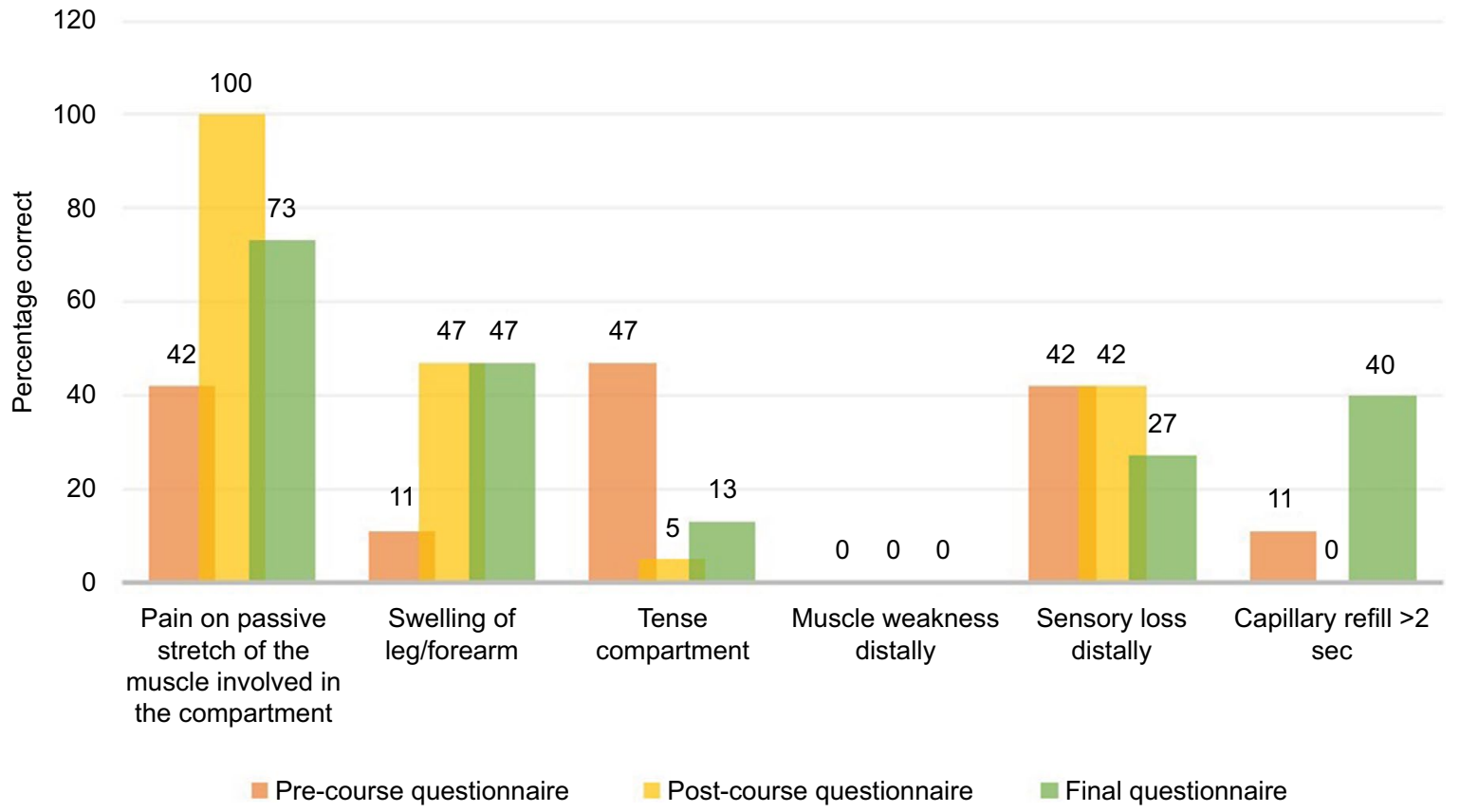

Figure 5 Question 4: The two most important signs of compartment syndrome include? Note: Correct answers: pain on passive stretch and sensory loss distally.

ACS in proximal humerus fractures. The implementation of the ward-based protocol specifically highlights "who is at risk", naming tibial and forearm fractures as common sites. Therefore, at 4 months, a significant improvement in recognition of elbow/forearm fractures as a risk factor for ACS was seen (93\%). Most nurses were aware that pain out of proportion to injury is the most important clinical symptom for diagnosing ACS.

The RCN guideline has pictorial depictions on performing passive movements of the fingers and toes. This may account for a high response rate for selecting pain on passive stretch (100\% post course and $73 \%$ at 4 months). Despite 
Immediate response after suspecting compartment syndrome should be (select 1):

\section{0}

\section{3}

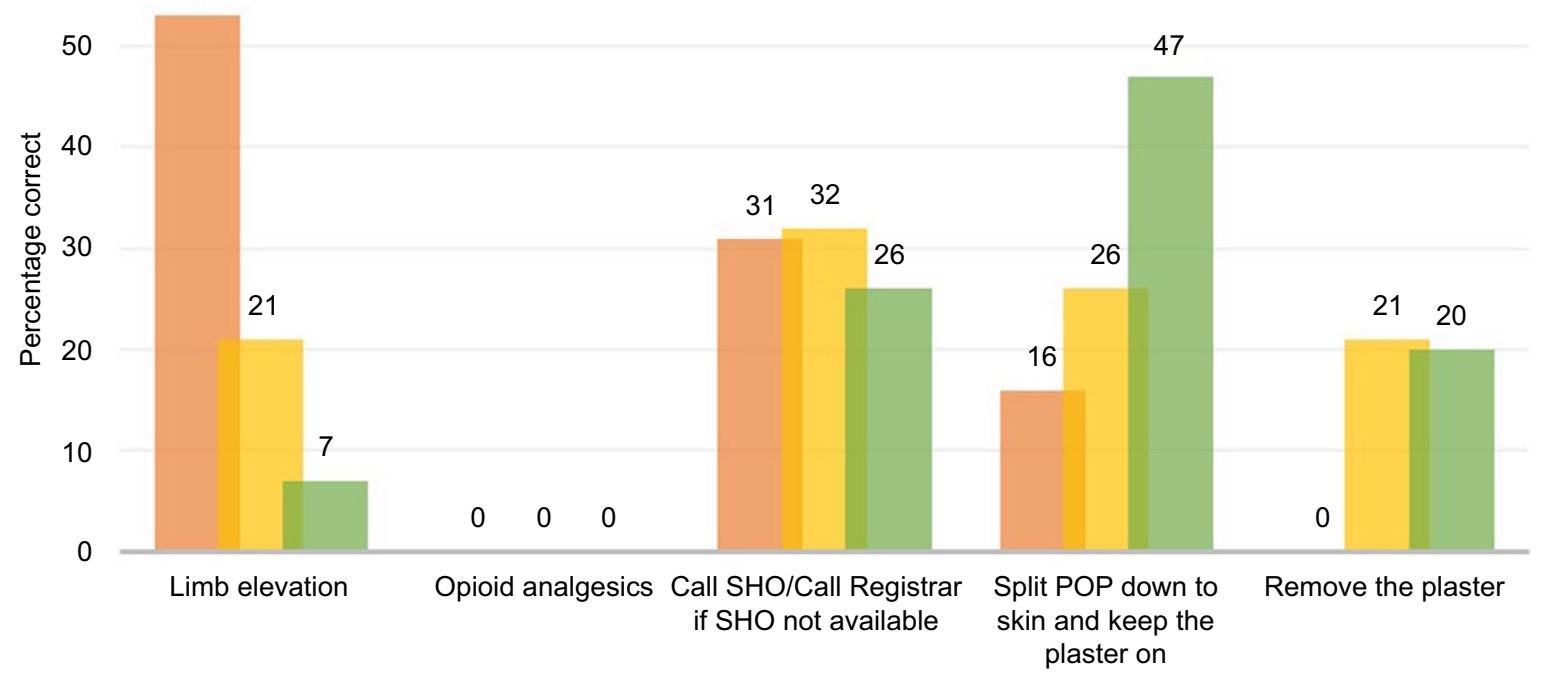

— Pre-course questionnaire $\quad$ Post-course questionnaire $\quad$ Final questionnaire

Figure 6 Question 5: Immediate response after suspecting compartment syndrome should be?

Note: Correct answer: split POP down to skin and keep the plaster on.

Abbreviation: POP, Plaster of Paris.

The most dreaded complication of compartment syndrome is (select 1):

\section{0}

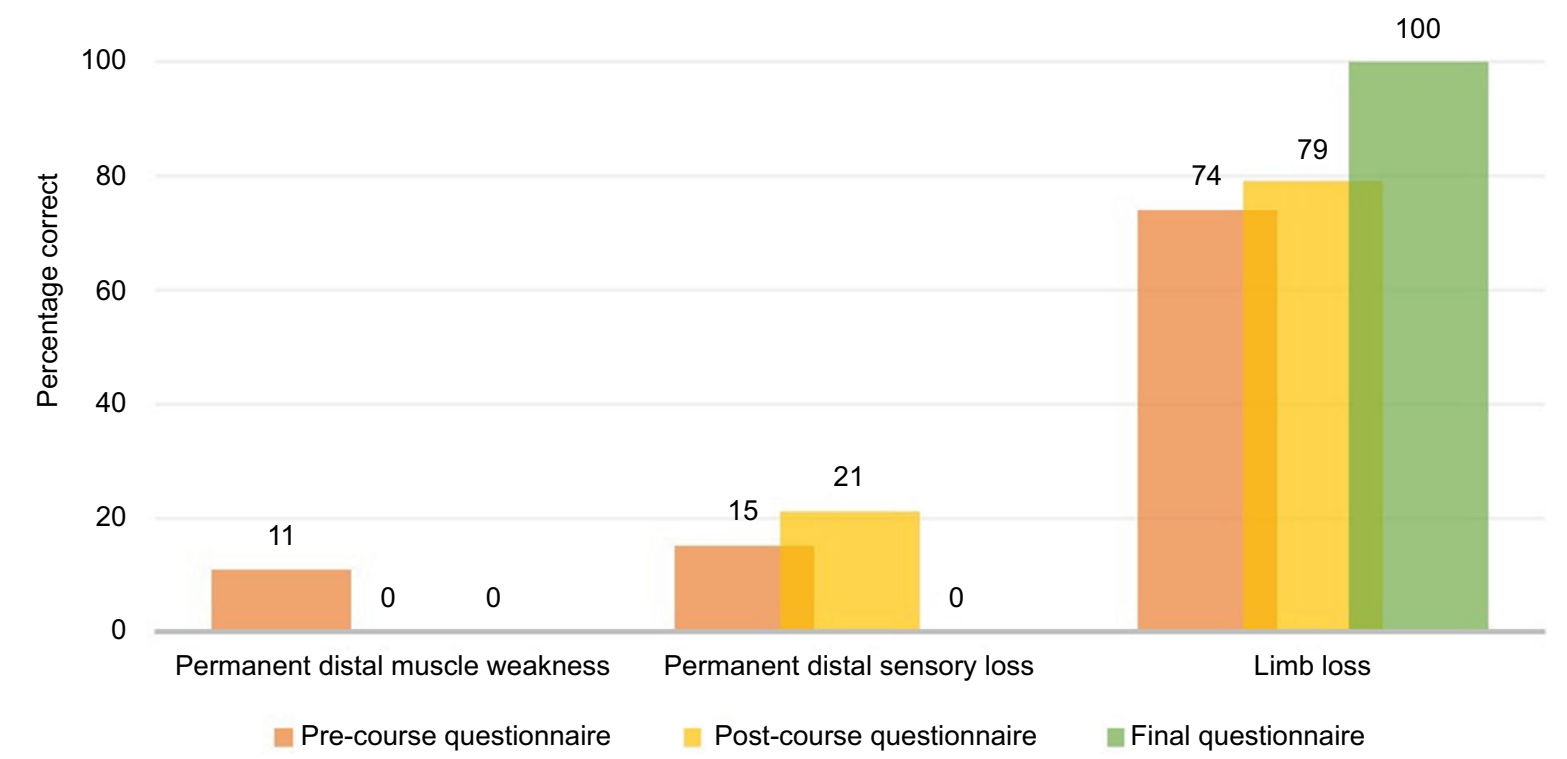

Figure 7 Question 6: The most dreaded complication of compartment syndrome is? Note: Correct answer: limb loss.

the lecture and implementation of the protocol, nursing staff were still unaware of distal sensory loss as an early sign. However, there is much debate regarding sensory loss as an early sign for ACS. Early work by Mubarak et a ${ }^{16}$ stated that sensory deficit is the most reliable physical finding of ACS. Similarly, McQueen et $\mathrm{al}^{2}$ reported that "sensory symptoms and signs are often the first indication of nerve ischaemia". There are several other studies emphasizing sensory loss as an early feature of compartment syndrome in the leg. ${ }^{10,11,17} \mathrm{~A}$ study by Gelberman et al ${ }^{18}$ focusing 
on ACS of the forearm highlighted that loss of two-point discrimination was a reliable indicator differentiating ACS from raised intracompartment pressure alone. ACS is still primarily a clinical diagnosis, and the absence of sensory symptoms should not delay surgical intervention but rather the presence of the sign can aid diagnosis.

Our results showed significant variability in the immediate response to suspecting compartment syndrome. Limb elevation would be counterproductive in ACS, and despite this being explained in the lecture was still selected by $21 \%$ of the participants. Only $26 \%$ of the participants correctly selected splitting the plaster, after the lecture. The ward-based protocol specifically states "split the POP down to skin (only split, do not remove)". It also states that the limb should be maintained at the heart level. Despite this written information, only $47 \%$ of the participants at 4 months selected physically intervening and splitting the plaster. In all, 26\% of the participants opted for a "safe" option of calling the on-call doctor and $20 \%$ still incorrectly selected complete plaster removal. Answers to this aspect of the questionnaire were quite disappointing and thus highlight the need for continuous learning for the ONs and TNPs so that appropriate ward-based interventions can occur.

A majority of the nurses involved in this audit had little or no prior clinical experience in the management of patients with ACS.

The above pre-course findings highlighted gaps in the core knowledge of recognizing and intervening in patients with compartment syndrome. The audit interventions (targeted lecture and early escalation protocol) attempted to address this deficit and do show some areas of improvement. At 4 months, notable improvement was seen in most areas; however, in certain areas, e.g., recognition of early signs and attempting early physical interventions were still lacking and would require further improvement. With the reduction in training hours for both nursing and medical staff, as well as it being a dynamic, changing workforce, special attention needs to be granted to topics given areas of limited exposure such as ACS. This highlights the need for continuous learning through lecture-based interventions and updating of core knowledge and skills every few months. This would be particularly pertinent to new orthopedic nursing staff and locum staff involved with orthopedic departments as they would undoubtedly be the first port of call for patients with suspected ACS.

While compartment measurements may be deemed out of their remits and scope of practice, the staff should all be able to clinically assess high-risk patients and attempt early intervention when there is a high index of suspicion for ACS. Early escalation to the on-call team is perfectly acceptable during diagnostic uncertainty.

\section{Conclusion}

ACS is a true surgical emergency requiring early diagnosis, early immediate intervention and subsequent definitive treatment via fasciotomy and decompression of all tissues within the affected compartment.

All medical staff working within a trauma and orthopedic setting should aim to enhance their baseline knowledge by being aware of common sites, early signs and symptoms, interventions and escalation plans. This would enable most effective recognition of the condition, significantly improving patient care. A delay in diagnosis and management will inevitably lead to a prolonged period of inadequate tissue perfusion and subsequently result in a poor outcome for the patient.

Our audit highlights that simple, lecture-based interventions allow staff to be kept up to date with current practices and provide a platform to build on for newer staff members. Similarly, having printed protocols on the wards acts as visual aide-memoires that could easily be used as a reference tool for diagnosis and for providing an escalation plan if necessary.

This audit also emphasizes the need for continuous teaching and training for ONs and TNPs to improve awareness and early diagnosis of the condition.

\section{Disclosure}

The authors report no conflicts of interest in this work.

\section{References}

1. Theerachai A, Surapong A, George B, et al. [homepage on the Internet]. AO Foundation Special Considerations - Compartment Syndrome. Available from: https://www2.aofoundation.org/. Accessed May 4, 2017.

2. McQueen MM, Christie J, Court-Brown CM. Acute compartment syndrome in tibial diaphyseal fractures. J Bone Joint Surg Br. 1996;78(1):95-98.

3. Ashton $\mathrm{H}$. Critical closing pressure in human peripheral vascular beds. Clin Sci. 1962;22:79-87.

4. Ashton H. The effect of increased tissue pressure on blood flow. Clin Orthop Relat Res. 1975;113:15-26.

5. McQueen MM, Court-Brown CM. Compartment monitoring in tibial fractures. The pressure threshold for decompression. J Bone Joint Surg Br. 1996;78(1):99-104.

6. Colton $\mathrm{C}$ [webpage on the Internet]. AO Foundation - Compartment Syndrome; 2016; v1.0 2016-11-22. Available from: https:// www2.aofoundation.org/wps/portal/surgery? showPage=redfix \& bone $=$ Tibia\&segment $=$ Distal $\&$ classification $=43-$ Special $\% 20$ conside rations\&treatment $=\&$ method $=$ Special $\% 20$ considerations $\&$ implant stype $=$ Complications\&approach $=\&$ redfix_url $=1341905827987$ \&Language=en - stepUnit-1. Accessed May 4, 2017. 
7. McQuillan WM, Nolan B. Ischaemia complicating injury. A report of thirty-seven cases. J Bone Joint Surg Br. 1968;50(3):482-492.

8. McQueen MM, Gaston P, Court-Brown CM. Acute compartment syndrome. Who is at risk? J Bone Joint Surg Br. 2000;82(2):200-203.

9. Rorabeck $\mathrm{CH}$. The treatment of compartment syndromes of the leg. $J$ Bone Joint Surg Br. 1984;66(1):93-97.

10. DeLee JC, Stiehl JB. Open tibia fracture with compartment syndrome. Clin Orthop Relat Res. 1981;160:175-184.

11. Matsen FA, Clawson DK. The deep posterior compartmental syndrome of the leg. J Bone Joint Surg Am. 1975;57(1):34-39.

12. Mithöfer K, Lhowe DW, Vrahas MS, Altman DT, Altman GT. Clinical spectrum of acute compartment syndrome of the thigh and its relation to associated injuries. Clin Orthop Relat Res. 2004;425:223-229.

13. Mubarak SJ, Wilton NC. Compartment syndromes and epidural analgesia. J Pediatr Orthop. 1997;17(3):282-284.
14. BOA. BOAST 10: Diagnosis and Management of Compartment Syndrome of the Limbs; 2016. Available from: https://www.boa.ac.uk/ wp-content/uploads/2014/12/BOAST-10.pdf. Accessed May 4, 2017.

15. RCN [webpage on the Internet]. Acute Limb Compartment Syndrome Observation Chart; 2016. Available from: https://www.ren.org.uk/ professional-development/publications/pub-005457. Accessed May 4, 2017.

16. Mubarak SJ, Owen CA, Hargens AR, Garetto LP, Akeson WH. Acute compartment syndromes: diagnosis and treatment with the aid of the wick catheter. J Bone Joint Surg Am. 1978;60(8):1091-1095.

17. Gulli B, Templeman D. Compartment syndrome of the lower extremity. Orthop Clin North Am. 1994;25(4):677-684.

18. Gelberman RH, Garfin SR, Hergenroeder PT, Mubarak SJ, Menon J. Compartment syndromes of the forearm: diagnosis and treatment. Clin Orthop Relat Res. 1981;161:252-261.

\section{Clinical Audit}

\section{Publish your work in this journal}

Clinical Audit is an international, peer-reviewed, open access journal focusing on the processes and outcomes of clinical audit in any area of healthcare. All aspects of patient care are addressed within the journal and practitioners from all disciplines are invited to submit their work. Areas covered include: Publication of audits; How an audit has changed practice;

Submit your manuscript here: https://www.dovepress.com/clinical-audit-journal

\section{Dovepress}

Practical tips on how to do audits and to avoid pitfalls; How audits have changed patient care; Calls and justifications for new audits. The manuscript management system is completely online and includes a very quick and fair peer-review system, which is all easy to use. Visit http://www.dovepress. com/testimonials.php to read real quotes from published authors. 